\title{
Fibre-based post systems: a review of the literature
}

\author{
Fibre-based post systems: a review

\section{G. Bateman, D. N. J. Ricketts and W. P. Saunders Br Dent J 2003; 195: 43-48}

\section{Objectives}

This article presents a review of published literature examining fibre-based endodontic post systems.

\section{Data sources}

A MEDLINE search was carried out for any articles in dental journals pertaining to fibre-based post systems. Wherever possible articles cited were obtained from the journals and where this was not possible abstracts were obtained. Where no abstract was available the article was not considered for evaluation.

\section{Data extraction}

Articles were reviewed by a single observer and subject to meeting inclusion criteria were included in the review. Fifty-nine articles were considered suitable for inclusion.

Data synthesis

Articles were divided into categories and a subjective description of the articles was made.

\section{Conclusions}

Review indicated that (1) most published literature on fibre-based posts took the form of laboratory analyses; (2) evidence for carbon-fibre posts far exceeds that for quartz-fibre posts; (3) laboratory evidence was contradictory and could not be used to inform practice reliably; (4) few clinical studies have been carried out though these have suggested fibre based posts may be clinically appropriate for restoration of the endodontically treated tooth; and (5) controlled prospective clinical trials evaluating fibre-based posts should be undertaken to inform use for clinical practice.

\section{IN BRIEF}

- Most published literature on fibre-based posts take the form of laboratory analyses.

- Evidence for carbon-fibre posts far exceeds that for quartz-fibre posts and further investigation of these posts is necessary before these can be recommended for routine use.

- Laboratory evidence was contradictory and may not be used to reliably inform practice.

- Few clinical studies have been carried out though these have suggested fibre-based posts may be clinically appropriate for restoration of the endodontically treated tooth.

- Controlled prospective clinical trials evaluating fibre-based posts should be undertaken before adoption in clinical practice.

\section{COMMENT}

In 1989, the French company RTD produced the first fibre-composite root canal post. In the next five years, very little was reported about this novel use of a fibre-reinforced material. It is only in the latter half of the 1990s that much interest has been shown in these new rivals to metal posts. Over this period, there has been a huge number of publications produced but also a range of different fibre posts introduced by many manufacturers. Differences in their resin chemistry, fibre composition, manufacturing processes and external geometry have resulted in an heterogeneous family of posts. What may be true of one post may therefore not be true for another, making the extrapolation of the research data to clinical practice more complicated. A further complication which affects many prospective clinical studies on materials is that the same material is often not in production at the end of a meaningful trial period.

This thorough review of published literature gives an appraisal of what has been discovered to date. The conclusions which the authors draw are justified and are therefore rather disappointing. They show that, despite the amount and variety of investigations which have been undertaken, conflicts in laboratory data and the limitations of the clinical studies performed hardly constitutes an evidence base for sound clinical practice. Such reviews all too commonly reveal the inadequacies of dental research. It is also worth remembering that, although this review concerns itself with information about only the fibre post, in the clinical situation in which they are used, the post is only one element of a complex restorative system including the root, the lute, the core, and the crown restoration. ${ }^{1}$ Separation of one element from the others is difficult, and may be misleading, particularly in clinical studies. For example, it has been recognized for some time that the design of the crown, particularly the provision of a ferrule, has an overriding influence on the outcome of crowns on root-filled teeth. ${ }^{2}$

Fibre reinforced posts were introduced based on two premises; that posts should not be more rigid than dentine, and that fibre posts have an elastic modulus similar to dentine. ${ }^{3}$ There is no consensus opinion or evidence regarding these concepts. ${ }^{4}$ However, despite the lack of this fundamental information, research continues.

Researchers need to provide practitioners with better quality evidence, from more meaningful laboratory tests, and in particular, with well controlled clinical trials as suggested by the authors of this review.

Dominic A. Stewardson, Clinical Lecturer, Conservative Dentistry, University of Birmingham School of Dentistry doi:10.1038/sj.bdj.4810307

1. Smith C T, Schuman N, Wasson W. Biomechanical criteria for evaluating prefabricated post-and-core systems; A guide for the restorative dentist. Quintessence Int 1998:29:305-312.

2. Gelfand $M$, Goldman $M$, Sunderman $E J$. Effect of complete veneer crowns on the compressive strength of endodontically treated posterior teeth. J Prosthet Dent 1984 52: 635-638.

3. Duret B, Reynaud M, Duret F. A new concept of corono-radicular reconstruction: the Composipost. Chir Dent Fr 1990; 60: 69-77.

4. Asmussen E, Peutzfeldt A, Heitmann T. Stiffness, elastic limit, and strength of newer types of endodontic posts. J Dent 1999; 27: 275-278. 